\title{
Growth of Dimension in Complete Simple Games
}

\author{
Liam O'Dwyer and Arkadii Slinko \\ The University of Auckland, New Zealand
}

\begin{abstract}
The concept of dimension in simple games was introduced by Taylor and Zwicker (1993) as a measure of the remoteness of a given game from a weighted game. They demonstrated that the dimension of a simple game can grow exponentially in the number of players. However, the problem of worst-case growth of the dimension in complete games was left open. Freixas and Puente (2008) showed that complete games of arbitrary dimension exist and, in particular, their examples demonstrate that the worst-case growth of dimension in complete games is at least linear. In this paper, using a novel technique of Kurz and Napel (2015), we demonstrate that the growth of dimension in complete games can also be exponential in the number of players.
\end{abstract}

Keywords:

simple game, complete simple game, dimension, boolean dimension

\section{Introduction}

The past two decades have witnessed an explosion of interest in computational and representational issues related to coalitional games (see, e.g., Deng and Papadimitriou (1994); Ieong and Shoham (2005)) and simple games, in particular (see, e.g., Delneko and Woeginger (2006); Elkind et al. (2008); Faliszewski et al. (2009)). Simple games were introduced in their present form by von Neumann and Morgenstern (1944) for applications in economics but found a wide range of applications across several disciplines. In particular, simple games are used to model decision making in committees $(\mathrm{Pe}-$ leg, 2002), reliability of real life systems made from unreliable components 
(Ramamurthy, 2012) and McCulloch-Pitts units in threshold logic (Muroga, 1971).

A simple game consists of a finite set of players and a set of winning coalitions that satisfies the monotone property asserting that all supersets of a winning coalition are also winning. One of the most important classes of simple games is the class of weighted simple games. In a weighted simple game every player is assigned a non-negative real weight so that a coalition is winning if the total weight of its players is at least some predetermined threshold. From the computational perspective weighted games are especially important since they can be succinctly represented by a finite sequence of integers (Freixas, 1997). If we allow the weights and the threshold to be vector-valued, then every simple game becomes weighted (Taylor and Zwicker, 1993) and the smallest dimension of vectors which makes this representation possible is called the dimension of the game.

Taylor and Zwicker (1999) demonstrated that the dimension of simple games can grow exponentially in the number of players. Recently Olsen et al. (2016) established the exact nature of this growth which is $2^{n-o(n)}$, where $n$ is the number of players.

Another important class of simple games is the class of complete games introduces by Carreras and Freixas (1996). In a complete game it is always possible to say which player among any two players is at least as desirable (as a coalition partner) as another one and, moreover, this desirability relation is a total order. This is a much broader class of games than weighted games which includes, for example, conjunctive and disjunctive hierarchical games which appear as the access structures of popular secret sharing schemes (Simmons, 1990; Tassa, 2007). Both disjunctive and conjunctive hierarchical games are seldom weighted (Gvozdeva et al., 2013) or even roughly weighted (Hameed and Slinko, 2015).

Freixas and Puente (2008) studied conjunctive hierarchical games (under the name of games with a minimum) and found that their dimension grows linearly in the number of players and asked whether or not in the class of complete games the dimension can grow polynomially or even exponentially. In the present paper we show that the growth of dimension of disjunctive hierarchical games, which are complete, is exponential in the number of players. This, in particular, fully answers the question of Freixas and Puente.

To obtain the aforementioned result we had to find a class of complete games whose dimension grows very fast. We found this growth in the class of disjunctive hierarchical games. We also turned our attention to conjunctive 
games for two reasons. Firstly, we fixed the gap in the aforementioned result of Freixas and Puente and also removed an unnesessary requirement of absence of veto and dummy players. The gap in their theorem is non-trivial and goes to the heart of the definition of dimension. In a way, this definition is less nice than Freixas and Puente thought. We devoted Section 4 for clarification of this problem.

\section{Preliminaries}

\section{Simple Games.}

Let $P$ be a set consisting of $n$ players. For convenience $P$ can be taken to be $[n]=\{1,2, \ldots, n\}$.

Definition 1. A simple game is a pair $G=(P, W)$, where $W$ is a subset of the power set $2^{P}$ which satisfies the monotonicity condition:

$$
\text { if } X \in W \text { and } X \subset Y \subseteq P \text {, then } Y \in W \text {. }
$$

Elements of the set $W$ are called winning coalitions. We also define the set $L=2^{P} \backslash W$ and call elements of this set losing coalitions. A winning coalition is said to be minimal if every proper subset of it is a losing coalition. A losing coalition is said to be maximal if every proper superset of it is winning. Due to monotonicity, every simple game is fully determined by the set of its minimal winning coalitions $W_{\min }$ or the set of maximal losing coalitions $L_{\max }$.

\section{Weighted Simple Games and Criteria of Weightedness.}

Definition 2. A simple game $G$ is called a weighted (majority) game if there exist non-negative reals $w_{1}, \ldots, w_{n}$, and a positive real number $q$, called the quota, such that $X \in W$ iff $\sum_{i \in X} w_{i} \geq q$. Such a game is denoted $\left[q ; w_{1}, \ldots, w_{n}\right]$. We also call $\left[q ; w_{1}, \ldots, w_{n}\right]$ a voting representation for $G$.

Example 1. Let now $n=2 k-1$ be odd and $W$ be all subsets of $P$ of cardinality $k$ or greater. There are exactly $2^{n-1}$ elements in $W$. This game is called the simple majority voting game. It is weighted and $[k ; 1,1, \ldots, 1]$ is its voting representation.

A more interesting example is 
Example 2. The UN Security Council consists of five permanent and 10 non-permanent members (which are sovereign states). A passage requires approval of at least nine countries, subject to a veto by any one of the permanent members. This is a weighted simple game with a voting representation

$$
[39 ; 7,7,7,7,7,1,1,1,1,1,1,1,1,1,1] .
$$

A sequence of coalitions

$$
\mathcal{T}=\left(X_{1}, \ldots, X_{j} ; Y_{1}, \ldots, Y_{j}\right)
$$

of simple game $G$ is a trading transform of length $j$ if the coalitions $X_{1}, \ldots, X_{j}$ can be converted into the coalitions $Y_{1}, \ldots, Y_{j}$ by rearranging players. It can also be expressed as

$$
\left|\left\{i: a \in X_{i}\right\}\right|=\left|\left\{i: a \in Y_{i}\right\}\right| \quad \text { for all } a \in P .
$$

A trading transform $\mathcal{T}$ is called a certificate of non-weightedness for $G$ if $X_{1}, \ldots, X_{j}$ are winning in $G$ and $Y_{1}, \ldots, Y_{j}$ are losing. The absence of certificates of non-weightedness of any length is a necessary and sufficient condition of weightedness of the game $G$ (Elgot, 1961; Taylor and Zwicker, 1999).

A more general class of games that we will touch upon is the class of roughly weighted games (Gvozdeva et al., 2013).

Definition 3. A simple game $G$ is called roughly weighted if there exist nonnegative real numbers $w_{1}, \ldots, w_{n}$ and a real number $q$, called the quota, not all equal to zero, such that for a coalition $X \in 2^{P}$ the condition $\sum_{i \in X} w_{i}<q$ implies $X$ is losing, and $\sum_{i \in X} w_{i}>q$ implies $X$ is winning.

Weighted games and a roughly weighted games both have a system of weights and a threshold. The difference is the treatment of coalitions on the threshold. In the weighted case all of them are winning and in the roughly weighted case both winning and losing coalitions can occur.

Finally a few words about duality.

Let $G=(P, W)$ be a simple game and $L$ be the set of its losing coalitions. We define the game $G^{*}=\left(P, W^{*}\right)$ dual to $G$ by setting

$$
W^{*}=\{P \backslash X \mid X \in L\},
$$

i.e., the winning coalitions of $G^{*}$ are complements to the losing coalitions of $G$. 


\section{Complete and Hierarchical Simple Games.}

Given a simple game $G=(P, W)$, after Isbell (1958), we define a relation $\succeq_{G}$ on $P$ by setting $i \succeq_{G} j$ if for every set $X \subseteq P$ not containing $i$ and $j$

$$
X \cup\{j\} \in W \Longrightarrow X \cup\{i\} \in W .
$$

In such a case we will say that $i$ is at least as desirable (as a coalition partner) as $j$. This relation is reflexive and transitive but not always complete (total) (e.g., see Carreras and Freixas (1996)). The corresponding equivalence relation on $[n]$ will be denoted $\sim_{G}$ and the strict desirability relation as $\succ_{G}$. If this can cause no confusion we will omit the subscript $G$.

Definition 4. A game whose desirability relation is complete is called complete.

Example 3. Any weighted game is complete.

Later we will have more examples. Complete simple games are a very natural generalisation of weighted games. This class is much larger, however, so measures of non-weightedness, e.g., the dimension, for such games are important and interesting.

In a complete game $G=(P, W)$ the set of players $P$ is partitioned into equivalence classes $P=P_{1} \cup \ldots \cup P_{m}$ with respect to $\sim_{G}$. Without loss of generality we will consider that

$$
P_{1} \succ_{G} P_{2} \succ_{G} \ldots \succ_{G} P_{m}
$$

Such game $G$ is called $m$-partite.

Any coalition $X \subseteq P$ defines a multiset $\left\{1^{\ell_{1}}, \ldots, m^{\ell_{m}}\right\}$, where $\ell_{i}$ is the number of elements from $P_{i}$ in $X$. Due to completeness, the status of a coalition $X$, i.e., whether it is winning or losing, can be deduced from this multiset. The multisets corresponding to winning coalitions will be called models of winning coalitions. We can also define models of losing coalitions, respectively.

In a complete game $G=(P, W)$ a winning coalition $X$ is shift-minimal if any coalition $(X \backslash\{i\}) \cup\{j\}$ is losing for any $i \in X$ and $j \notin X$ such that $i \succ_{G} j$, i.e., it ceases to be winning after any replacement of its player with a less desirable one. A losing coalition $Y$ is called shift-maximal if it becomes winning after a replacement of any player with a more desirable player. A 
complete simple game is fully defined by the set of its shift-minimal winning coalitions or a set of its shift-maximal losing coalitions.

Suppose now that the set of players $P$ is partitioned into $m$ disjoint subsets $P=\cup_{i=1}^{m} P_{i}$ and let $k_{1}<k_{2}<\ldots<k_{m}$ be a sequence of positive integers. Let $\mathbf{k}=\left(k_{1}, \ldots, k_{m}\right)$. Then we define the game $H=H_{\exists}(P, \mathbf{k})$ by setting the set of winning coalitions to be

$$
W_{\exists}=\left\{X \in 2^{P} \mid \exists i\left(\left|X \cap\left(\cup_{j=1}^{i} P_{i}\right)\right| \geq k_{i}\right)\right\} .
$$

Such a game is called a disjunctive hierarchical game. It has $m$ thresholds and one of them must be reached for the coalition to be winning.

Suppose now that the set of players $P$ is partitioned into $m$ disjoint subsets $P=\cup_{i=1}^{m} P_{i}$, and let $k_{1}<\ldots<k_{m-1} \leq k_{m}$ be a sequence of positive integers. Then we define the game $H_{\forall}(P, \mathbf{k})$ by setting the set of its winning coalitions to be

$$
W_{\forall}=\left\{X \in 2^{P} \mid \forall i\left(\left|X \cap\left(\cup_{j=1}^{i} P_{i}\right)\right| \geq k_{i}\right)\right\} .
$$

Such a game is called a conjunctive hierarchical game. It has $m$ thresholds and all of them must be reached for the coalition to be winning.

Both classes of hierarchical games are complete. Gvozdeva et al. (2013) give a sufficient and necessary conditions for the game $H_{\forall}(P, \mathbf{k})$ defined above to be truly $m$-partite ${ }^{1}$. We denote $\left|P_{i}\right|=n_{i}$, then the following two conditions jointly are necessary and sufficient:

$$
\begin{aligned}
k_{1} & \leq n_{1} \\
k_{i} & <k_{i-1}+n_{i}
\end{aligned}
$$

for every $i \in\{2, \ldots, m\}$. Moreover, Gvozdeva et al. (2013) showed that $G$ has veto players if and only if $k_{1}=n_{1}$, in which case $P_{1}$ is the set of veto players, and $G$ has dummy players if and only if $k_{m-1}=k_{m}$, in which case $P_{m}$ is the set of dummy players.

We note that these conditions imply

$$
k_{i} \leq n_{1}+\ldots+n_{i}-(i-1) .
$$

\footnotetext{
${ }^{1}$ For some combinations of parameters the number of equivalence classes may in fact be less than $m$.
} 
for all $i=1, \ldots, m$, and

$$
n_{i}>1 .
$$

for all $1<i<m$, moreover, it is also true for $i=m$ in the absence of dummy players. The first inequality follows from (4). In particular, it shows that the equation $k_{i}=n_{1}+\ldots+n_{i}$ can be satisfied only when $i=1$. The second also follows from (4) since

$$
n_{i}>k_{i}-k_{i-1} \geq 1
$$

(for $i=m$ this needs $k_{m} \neq k_{m-1}$, which means no dummies.

Lemma 1. Let $G=(P, W)$ be an m-partite simple game with $P=P_{1} \cup P_{2} \cup$ $\ldots \cup P_{m}$, where $P_{1}$ consists of veto players and $P_{m}$ of dummy players. Let $A=P_{1} \cup P_{m}$. Then the reduced game $G^{A}$ is defined on the set of players $P_{2} \cup \ldots \cup P_{m-1}$ and does not have veto or dummy players).

Proof. Due to Proposition 5 of Grozdeva et al. (2013) $G_{A}$ is an $(m-2)$ partite conjunctive hierarchical game with $\mathbf{n}^{\prime}=\left(n_{2}, \ldots, n_{m-1}\right)$ and with the vector of thresholds

$$
\mathbf{k}^{\prime}=\left(k_{2}-k_{1}, \ldots, k_{m-1}-k_{1}\right) .
$$

Since $n_{2}>k_{2}-k_{1}$ this game does not have veto players and since $k_{m-2}-k_{1}<$ $k_{m-1}-k_{1}$ it does not have dummies.

\section{Definition of the Dimension. The Criterion of Kurz and Napel.}

The dimension of a simple game $G$ is the minimum dimension of the vectors required to express it as a vector-weighted game (Taylor and Zwicker, 1993). That is, a simple game has dimension $k$ if it can be represented as a vector-weighted game with weights from $\mathbb{R}^{k}$, but not with weights from $\mathbb{R}^{\ell}$ for $\ell<k$. In practice it is more convenient to work with the following equivalent definition.

Let $G_{i}=\left(P, W_{i}\right), i=1, \ldots, n$, be simple games on the same set of players $P$. Then the intersection of these games is the simple game $G=$ $(P, W)$, where $W=W_{1} \cap W_{2} \cap \cdots \cap W_{n}$. In other words, a coalition is winning in $G$ if and only if it is winning in $G_{i}$ for each $i=1, \ldots, n$. We write $G=G_{1} \wedge \ldots \wedge G_{n}$ for reasons that will be revealed later. 
Definition 5. A simple game has dimension d if it can be represented as the intersection of $d$ weighted games but cannot be represented as the intersection of $\ell$ weighted games for $\ell<d$. We will denote the dimension of $G$ by $\operatorname{dim}(G)$.

Examples of games of dimension 2 include the United States Federal System and the procedure to amend the Canadian Constitution (Taylor and Zwicker, 1999). Freixas (2004) showed that the dimension of the European Union Council under the Nice rules had dimension 3. In a recent article Kurz and Napel (2015) have found that the revised voting rules of the Council of the European Union (EU Council) mean that a simple game representation of that voting body must have dimension at least 7 . This is significantly larger than that of any other known simple game that occurs in the real world.

To calculate the dimension of a game exactly is not an easy task. Deineko and Woeginger (2006) proved that the following problem is NP-hard: given $k$ weighted majority games on the same set of players, decide whether the dimension of their intersection is exactly $k$.

To bound the dimension of a game from above the following observation can be used (Taylor and Zwicker, 1999).

Proposition 1. The dimension of a simple game $G=(P, W)$ is at most the cardinality $\left|L_{\max }\right|$ of the set $L_{\max }$ of maximal losing coalitions of $G$.

To bound the dimension of a game from below we will use the following useful criterion, which is Observation 1 in Kurz and Napel (2015). It is so important that we call it a theorem.

Theorem 1. Let $G=(P, W)$ be a simple game, and let $S=\left\{Y_{1}, \ldots, Y_{k}\right\}$ be a set of losing coalitions such that for each pair $\left\{Y_{i}, Y_{j}\right\}$ with $i \neq j$, there is no weighted simple game for which every coalition in $W$ is winning but $Y_{i}$ and $Y_{j}$ are both losing. Then the dimension of $G$ is at least $|S|=k$.

Kurz and Napel refer to these elements of $S$ as pairwise incompatible. One way to use this theorem to prove that a simple game $G$ has dimension at least $k$ is to find, for every pair $\left\{Y_{i}, Y_{j}\right\} \subseteq S$, a certificate of non-weightedness $\left(X_{i, j}^{1}, X_{i, j}^{2} ; Y_{i}, Y_{j}\right)$, where $X_{i, j}^{1}$, and $X_{i, j}^{2}$ are both winning in $G$.

\section{The Main Results}

\section{Dimension of Disjunctive Hierarchical Games.}


Firstly, let us consider a non-weighted example of a disjunctive hierarchical game with the 'smallest' possible vector $\mathbf{k}$. This would be $\mathbf{k}=(2,4)$ (as in non-trivial cases we have $k_{1} \geq 2$ and the games with $\mathbf{k}=(2,3)$ are weighted (Gvozdeva et al., 2013)). Although not weighted, it is known (Gvozdeva et al., 2013), that the game with this set of parameters is always roughly weighted.

Proposition 2. Let $d \geq 2$ be a positive integer. Let $P=P_{0} \cup P_{1}$ with $\left|P_{0}\right|=d,\left|P_{1}\right|=2 d$, and $\mathbf{k}=(2,4)$. Then the disjunctive hierarchical game $H=H_{\exists}(P, \mathbf{k})$ has dimension at least $d$.

Proof. Let $P_{0}=\left\{a_{0}, \ldots, a_{d-1}\right\}$ and $P_{1}=\left\{b_{0}, \ldots, b_{2 d-1}\right\}$, define the sets $Y_{i}=\left\{a_{i}, b_{2 i}, b_{2 i+1}\right\}, i=0, \ldots, d-1$, and let $S=\left\{Y_{0}, \ldots, Y_{d-1}\right\}$. All coalitions from $S$ lose in $H$ since they have neither two players from $P_{1}$, nor four players in total. Then $S$ satisfies the conditions of the Kurz-Napel criterion since if $i \neq j$

$$
\left(\left\{a_{i}, a_{j}\right\},\left\{b_{2 i}, b_{2 i+1}, b_{2 j}, b_{2 j+1}\right\} ; Y_{i}, Y_{j}\right)
$$

is a certificate of non-weightedness for $H$ as the coalitions $X_{i, j}^{1}=\left\{a_{i}, a_{j}\right\}$ and $X_{i, j}^{2}=\left\{b_{2 i}, b_{2 i+1}, b_{2 j}, b_{2 j+1}\right\}$ are both winning (the first achieves the first threshold and the second achieves the second). By the criterion, the dimension of $H$ is at least $d$.

Thus we have the following result.

Theorem 2. There exist roughly weighted games of arbitrary large dimension.

Interestingly, we showed that we can get linear growth in the number of players without increasing the number of classes of equivalent players. If we start increasing both we will get a growth faster than linear.

Lemma 2. Let $P=P_{0} \cup P_{1} \cup \ldots \cup P_{m-1}$ with $\left|P_{0}\right|=k,\left|P_{1}\right|=\left|P_{2}\right|=\cdots=$ $\left|P_{m-1}\right|=2 k$ and $\mathbf{k}=(2,4,6, \ldots, 2 m)$. Then the disjunctive hierarchical simple game $H=H_{\exists}(P, \mathbf{k})$ has dimension d satisfying

$$
k^{m-1} \leq d \leq k^{m}(2 k-1)^{m-1} .
$$

Proof. Let $P_{0}=\left\{a_{1}, \ldots, a_{k}\right\}$ and denote by $p_{i}^{(j)}, j \in\{0, \ldots, 2 k-1\}$, the $j$ th player from part $P_{i}$. We will also denote $P_{i}^{(j)}=\left\{p_{i}^{(2 j)}, p_{i}^{(2 j+1)}\right\}$, where $j \in\{0, \ldots, k-1\}$ and $i \in[m]$. Then all coalitions of the form

$$
\left\{a_{i_{0}}\right\} \cup P_{1}^{\left(i_{1}\right)} \cup P_{2}^{\left(i_{2}\right)} \cup \cdots \cup P_{m-2}^{\left(i_{m-2}\right)} \cup P_{m-1}^{\left(i^{\prime}\right)},
$$


where $i^{\prime}=i_{0}+i_{1}+\ldots+i_{m-2}(\bmod k)$ will form the set $S$ to be used in the Kurz-Napel criterion. Firstly, we note that all the coalitions in $S$ are losing as no threshold is achieved. Let us show that any two of them are incompatible. Let

$$
\begin{aligned}
& Y_{1}=\left\{a_{i_{0}}\right\} \cup P_{1}^{\left(i_{1}\right)} \cup P_{2}^{\left(i_{2}\right)} \cup \cdots \cup P_{m-2}^{\left(i_{m-2}\right)} \cup P_{m-1}^{\left(i^{\prime}\right)}, \\
& Y_{2}=\left\{a_{j_{0}}\right\} \cup P_{1}^{\left(j_{1}\right)} \cup P_{2}^{\left(j_{2}\right)} \cup \cdots \cup P_{m-2}^{\left(j_{m-2}\right)} \cup P_{m-1}^{\left(j^{\prime}\right)},
\end{aligned}
$$

be two coalitions from $S$. There are two cases.

- If $i_{0}=j_{0}$, then there is at least one $\ell \in\{1, \ldots, m-2\}$ for which $i_{\ell} \neq j_{\ell}$ (otherwise the coalitions would be identical), so $P_{\ell}^{\left(i_{\ell}\right)}$ is disjoint from $P_{\ell}^{\left(j_{\ell}\right)}$. If $i_{r}=j_{r}$ for all $r \in\{1, \ldots, \ell-1, \ell+1, \ldots, m-2\}$, then $i^{\prime} \neq j^{\prime}$, thus we may assume that there exists also $r \in[m-1]$ such that $r \neq \ell$ and $i_{r} \neq j_{r}$. Without loss of generality assume that $\ell<r$. Then we get a certificate of non-weightedness

$$
\left(Y_{1} \cup\left\{p_{\ell}^{\left(2 j_{\ell}\right)}\right\} \backslash P_{r}^{\left(i_{r}\right)},\left(Y_{2} \backslash\left\{p_{\ell}^{\left(2 j_{\ell}\right)}\right\} \cup P_{r}^{\left(i_{r}\right)} ; Y_{1}, Y_{2}\right) .\right.
$$

by swapping one element $\left\{p_{\ell}^{\left(2 j_{\ell}\right)}\right\}$ of $Y_{2}$ for two elements of $P_{r}^{\left(i_{r}\right)}$ from $Y_{1}$. After the swap the first coalition will be winning since the $\ell$-th threshold is achieved and the second will be also winning since the $r$-th threshold is achieved.

- If $i_{0} \neq j_{0}$ but $i_{r}=j_{r}$ for all $r \in\{1, \ldots, m-2\}$, then $i^{\prime} \neq j^{\prime}$, hence we may assume that there exists $r \in[m-1]$ such that $i_{r} \neq j_{r}$. In this case we get a certificate of non-weightedness

$$
\left(Y_{1} \cup\left\{a_{j_{0}}\right\} \backslash P_{r}^{\left(i_{r}\right)},\left(Y_{2} \backslash\left\{a_{j_{0}}\right\} \cup P_{r}^{\left(i_{r}\right)} ; Y_{1}, Y_{2}\right)\right.
$$

by swapping $\left\{a_{j_{0}}\right\}$ and $P_{r}^{\left(i_{r}\right)}$.

Since $|S|=k^{m-1}$, by Theorem 1 , this means that the dimension of such a game is at least $k^{m-1}$.

The upper bound is easily calculated with the help of Proposition 1 taking in consideration that each maximal losing coalition consists of one member from $P_{0}$ and two members from each of the $P_{1}, \ldots, P_{n}$.

Theorem 3. In the class of disjunctive hierarchical games with bounded number of equivalence classes the worst-case growth of dimension is polynomial. 
Proof. If in (7) we fix $m$, then we have $(2 k+1) m$ voters which is linear in $k$ and the dimension which is polynomial in $k$. Hence the dimension growth is polynomial of degree $m$.

Theorem 4. In the class of disjunctive hierarchical games with bounded number of players in equivalence classes the worst-case growth of dimension is exponential.

Proof. If in (7) we fix $k$, then we have $(2 k+1) m$ voters which is linear in $m$ and the dimension is exponential in $m$. Hence the dimension growth is exponential in $m$.

These results answer directly the question from Freixas and Puente (2008) about possibility of a polynomial or exponential growth in complete simple games.

\section{Dimension of Conjunctive Hierarchical Games.}

Freixas and Puente (2008) studied a class of games, that they called games with minimum, which, as was proved in Gvozdeva et al. (2013), is nothing other than the class of conjunctive hierarchical games. The theorem they formulated state that the dimension $d$ of an $m$-partite conjunctive hierarchical game without veto or dummy players satisfies the inequalities $\left\lceil\frac{m}{2}\right\rceil \leq d \leq m$. However, we have to reprove the lower bound in this theorem due to two reasons: 1) the gap in their proof which will be explained in the next section after rectification of the concept of dimension; 2) the unnecessary requirement of having no dummies or vetoers.

But, firstly, we will give a simple proof of the upper bound as well getting rid of unnecessary requirement of having no dummies or vetoers.

Proposition 3. The dimension d of an m-partite conjunctive hierarchical game is at most $m$.

Proof. Let $H=H_{\forall}(P, \mathbf{k})$, where $P=P_{1} \cup \ldots \cup P_{m}$ with $\mathbf{k}=\left(k_{1}, \ldots, k_{m}\right)$, and suppose the game is $m$-partite. Let us denote $n=|P|$. Let us define $m$ weight functions on $P$ by

$$
w_{s}(p)= \begin{cases}1, & \text { if } p \in P_{1} \cup \ldots \cup P_{s} \\ 0, & \text { if } p \in P_{s+1} \cup \ldots \cup P_{m}\end{cases}
$$

and $m$ thresholds $q_{s}=k_{s}, s \in[m]$. We define game $G_{s}$ on $P$ by the weight function $w_{s}$ and threshold $q_{s}$. It is clear that a coalition $X$ wins in $G_{s}$ if and only if $\left|X \cap\left(\cup_{j=1}^{s} P_{i}\right)\right| \geq k_{s}$, hence $H=G_{1} \wedge \ldots \wedge G_{m}$ and $d \leq m$. 
We will need the following lemma.

Lemma 3. Let $H=H_{\forall}(P, \mathbf{k})$ be m-partite conjunctive hierarchical game with $P=P_{1} \cup \cdots \cup P_{m}$ being the equivalence classes of $\sim_{H},\left|P_{i}\right|=n_{i}$ and $\mathbf{k}=\left(k_{1}, \ldots, k_{m}\right)$. Then, if no dummies present, any shift-maximal losing coalition of $H$ corresponds to one of the following $m$ models:

$$
\mathcal{M}_{i}=\left\{1^{a_{1}}, \ldots, i^{a_{i}},(i+1)^{n_{i+1}}, \ldots, m^{n_{m}}\right\} \quad(i=1, \ldots, m),
$$

where $a_{1}, \ldots, a_{i}$ satisfy the following conditions:

(i) $a_{1}+\ldots+a_{i}=k_{i}-1$;

(ii) If $0<a_{t}$ for $t \leq i$, then $a_{s}=n_{s}$ for all $s<t$;

(iii) $a_{i}<n_{i}$.

If dummies present, then the model $\mathcal{M}_{m}$ does not exist and we have only $m-1$ models.

Proof. Suppose the last $m$ th level does not consist of dummies. Suppose that a maximal losing coalition $X$ fails the $i$ th condition, i.e., in the first $i$ levels there are no $k_{i}$ elements, that is, $a_{1}+\ldots+a_{i}<k_{i}$. Due to (5) we may assume that $a_{1}+\ldots+a_{i}=k_{i}-1$. Since the $i$ th threshold is already violated, we can include all elements of $P_{i+1} \cup \cdots \cup P_{m}$ without making this coalition winning. If for $i \leq t$ we have $a_{t}>0$ but $a_{t-1}<n_{t-1}$, then we can replace one element of level $t$ with element of level $t-1$ without making coalition winning. As $X$ was shift-maximal, this is not possible. If the $m$ th level consists of dummies, then $k_{m-1}=k_{m}$ so it is impossible to fail just one last threshold. Finally, if $a_{i}=n_{i}$, then by (ii) we have $a_{s}=n_{s}$ for all $s=1, \ldots, i-1$, in which case $X=P$.

We illustrate this lemma with an example.

Example 4. Let us consider the game $H_{\forall}(P, \mathbf{k})$, where $\left|P_{1}\right|=\left|P_{2}\right|=\left|P_{3}\right|=$ 4 , and $\mathbf{k}=(2,4,7)$. Then there are three models of shift-maximal losing coalitions:

$$
\left\{1,2^{4}, 3^{4}\right\}, \quad\left\{1^{3}, 3^{4}\right\}, \quad\left\{1^{4}, 2^{2}\right\} .
$$

The first type of coalitions fail the first threshold, the second type of coalitions fail the second threshold and the third type of coalitions fail the third threshold. 
We can now prove the main result of this subsection.

Theorem 5. The dimension d of an m-partite conjunctive hierarchical game $H$ satisfies $\left\lceil\frac{m-1}{2}\right\rceil \leq d \leq m$. If $H$ has no dummy players, then $\left\lceil\frac{m}{2}\right\rceil \leq d \leq m$.

Proof. It is easy to see that adding a level of dummy players does not change the dimension of the game so we may assume that $H$ has no dummy players. To prove the lower bound we assume that there are $\ell<\left\lceil\frac{m}{2}\right\rceil$ weighted games $H_{1}, \ldots, H_{\ell}$ such that $H=H_{1} \wedge \ldots \wedge H_{\ell}$. Then, in each game $H_{i}$, all winning coalitions of $H$ are winning and if a coalition is losing, then it is losing in one of the $H_{i}$.

So there are $\ell<m / 2$ games $H_{1}, \ldots, H_{\ell}$ but by Lemma 3 we have $m$ shift-maximal losing coalitions. Hence, due to the pigeonhole principle there exist an index $i$ such that in $H_{i}$ at least three shift-maximal losing coalitions of $H$, belonging to different models, are losing in $H_{i}$. Then there are two coalitions among these, say $L_{1}$ and $L_{2}$, whose corresponding models, say $\mathcal{M}_{i}$ and $\mathcal{M}_{j}$, satisfy $i+2 \leq j$. Then

$$
\begin{aligned}
& L_{1}=C_{1} \cup \ldots \cup C_{i} \cup P_{i+1} \cup \ldots \cup P_{m}, \\
& L_{2}=D_{1} \cup \ldots \cup D_{j} \cup P_{j+1} \cup \ldots \cup P_{m},
\end{aligned}
$$

where $C_{i} \subseteq P_{i}, D_{i} \subseteq P_{i}$, moreover, by Lemma 3 (i) we have $\left|C_{1}\right|+\ldots+\left|C_{i}\right|=$ $k_{i}-1$ and $\left|D_{1}\right|+\ldots+\left|D_{j}\right|=k_{j}-1$ with $\left|C_{i}\right|<n_{i}$ and $\left|D_{j}\right|<n_{j}$. We note that Lemma 3 also implies that $\left|C_{s}\right| \leq\left|D_{s}\right|$ for $s \in[i]$.

Let $s \in[j]$ be the largest positive integer with $D_{s} \neq \emptyset$. Then $\left|D_{1}\right|+\ldots+$ $\left|D_{s}\right|=\left|D_{1}\right|+\ldots+\left|D_{j}\right|=k_{j}-1$. Suppose $s \leq i$. As $k_{j}-1 \geq k_{j-1}>k_{i}$, we have $\left|C_{s}\right|<\left|D_{s}\right|$. If $s>i$, then $D_{i}=P_{i}$ and $\left|C_{i}\right|<\left|D_{i}\right|$ is also true. Thus, a transfer of a player, say $x$, from $D_{s}$ to $C_{s}$ in the first case and from $D_{i}$ to $C_{i}$ in the second, is possible. Let us do this transfer and, for simplicity, let us keep notation for these sets unchanged.

After the transfer we will have

$$
\left|D_{1}\right|+\ldots+\left|D_{j}\right|=k_{j}-2,
$$

i.e., the $j$ th threshold will become further from reach but let us show that for any $t \in[j-1]$ the $t$-th threshold is still reached for $L_{2} \backslash\{x\}$. If $D_{j-1}=\emptyset$, this is clear. If $D_{j-1} \neq \emptyset$, then by Lemma 3 (ii) we have $D_{r}=P_{r}$ for all $r \in[j-2]$. Due to (5) we see that only $(j-1)$ th threshold may be violated by $L_{2} \backslash\{x\}$. After the transfer we have, however, by (5)

$$
\left|D_{1}\right|+\ldots+\left|D_{j-1}\right|=n_{1}+\ldots+n_{j-1}-1 \geq k_{j-1}+(j-3) \geq k_{j-1}
$$


since $j \geq i+2 \geq 3$.

Now since $i<j-1$ we have $L_{1} \supset P_{j}$. If $D_{j-1}=P_{j-1}$, then by (5) and (9)

$$
n_{j}-\left|D_{j}\right|=n_{1}+\ldots+n_{j}-\left(k_{j}-2\right) \geq 2 \text {, }
$$

and by (6) there is a capacity to move two elements from $C_{j}$ to $D_{j}$. If $D_{j-1} \neq P_{j-1}$, then $D_{j}=\emptyset$ and again there is a capacity to move two elements from $C_{j}$ to $D_{j}$. Suppose the elements transferred are $y, z \in C_{j}$. This transfer will make $\left(L_{2} \backslash\{x\}\right) \cup\{y, z\}$ winning.

Let us now notice that for $L_{1}$ before the transfer we had

$$
\left|C_{1}\right|+\ldots+\left|C_{i}\right|+\left|P_{i+1}\right|+\ldots+\left|P_{r}\right| \geq k_{r}+(r-i)
$$

for any $r \geq j$. Indeed, by (4)

$$
\begin{aligned}
& \left|C_{1}\right|+\ldots+\left|C_{i}\right|+\left|P_{i+1}\right|+\ldots+\left|P_{r}\right|=k_{i}-1+\sum_{t=i+1}^{r} n_{t} \\
& \geq k_{i}-1+\sum_{t=i+1}^{r}\left(k_{t}-k_{t-1}-1\right)=k_{r}+(r-i) .
\end{aligned}
$$

This means that $\left(L_{1} \cup\{x\}\right) \backslash\{y, z\}$ is winning as well. We obtained a certificate of non-weightedness

$$
\left(\left(L_{1} \cup\{x\}\right) \backslash\{y, z\},\left(L_{2} \backslash\{x\}\right) \cup\{y, z\} ; L_{1}, L_{2}\right)
$$

which gives us a contradiction.

The only way we can grow the dimension in hierarchical conjunctive games is to increase the number $m$ of equivalence classes. This was not the case whith disjunctive hierarchical games.

Theorems 4 and 5 demonstrate that, inter alia, the dimension is not preserved under duality. Indeed, it is known (Gvozdeva et al., 2013) that the duality takes us from disjunctive hierarchical games to conjunctive ones and vice versa and their respective growth of dimension are very different.

\section{Codimension.}

A concept closely related to dimension is that of the codimension. 
Definition 6. The codimension of a simple game is the minimum number of weighted simple games whose union forms the given game. That is, the simple game $G=(P, W)$ has codimension $n$ if $W=W_{1} \cup \cdots \cup W_{n}$, where each of the games $\left(P, W_{1}\right), \ldots,\left(P, W_{n}\right)$ is weighted, and $W$ cannot be represented as the union of fewer than $n$ weighted games. We will denote the codimension of $G$ by $\operatorname{codim}(G)$.

This concept emerges in relation to the duality of games.

Theorem 6 (Freixas-Marciniak, 2009). If $G$ is a simple game, then

$$
\operatorname{codim}\left(G^{*}\right)=\operatorname{dim}(G)
$$

Proof. This is a simplification of Theorem 3.2(ii) in Freixas and Marciniak (2009), in which we take $\mathcal{C}$ to be the class of weighted simple games, along with their observation that this class is closed under duality.

Due to Freixas-Marciniak theorem we can extract some consequences from our results with respect to codimension.

Corollary 1. The codimension $d$ of an m-partite disjunctive hierarchical game satisfies $\left\lceil\frac{m}{2}\right\rceil-1 \leq d \leq m$.

Proof. Follows from Theorem 5 and the fact that the dual game to a disjunctive hierarchical game is conjunctive hierarchical (Gvozdeva et al., 2013).

Corollary 2. There is a sequence of conjunctive hierarchical games whose codimensions grow exponentially in the number of players.

\section{Problems with the concept of dimension}

There are several worrying properties of the concept of dimension. One of these can be observed from the results of this paper, namely, that the dimension is not preserved under duality. Indeed, in Gvozdeva et al. (2013) it was proved that the dual of a disjunctive hierarchical games is a conjunctive hierarchical games and vice versa. However, Theorems 4 and 5 show that the dimensions of games in these classes are very different. Another problem with this concept is illustrated in the following example. 
Motivating example.2 It shows that when one represents a complete game $G$ as an intersection of weighted games, it may be impossible to choose the weightings in a way that faithfully represents the desirability order $\succeq_{G}$ in $G$.

Let $P=P_{1} \cup P_{2}$ with $\left|P_{1}\right|=2$ and $\left|P_{2}\right|=5$. Consider a disjunctive hierarchical game $H=H_{\exists}(P, \mathbf{k})$ with $\mathbf{k}=(2,5)$. Frstly, let us consider $P$ as a multiset $\left\{1^{2}, 2^{5}\right\}$, i.e., consisting of two identical players of type 1 and five of type 2 . We ask if it is possible to find games $H_{1}, \ldots, H_{k}$, where in the $i$ th game weight $w_{i}(1)$ is assigned to all players of the first type, weight $\left.w_{i}(2)\right]$ to players of the second type and the threshold is $q_{i}$.

Considering classes of equivalent coalitions of $H$ as submultisets, we can graphically represent them as points in $\mathbb{R}$ representing the coalition $\left\{1^{x}, 2^{y}\right\}$ as point $(x, y)$. Thus, the minimal winning ones are $\left\{1^{2}\right\},\left\{1,2^{4}\right\},\left\{2^{5}\right\}$ will be represented as points $(2,0),(1,4),(0,5)$. We can graphically depict them on the following diagram where the area of winning coalitions is presented in grey.

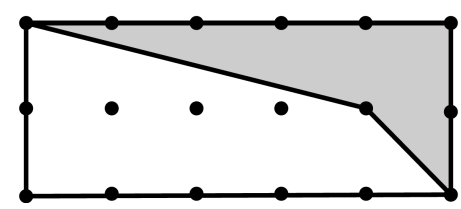

Figure 1: Vertical axis $x$ shows the number of players of the first type and horizontal axis $y$ of the second.

If it was possible to find weight functions $w_{1}, w_{2}$ and thresholds $q_{1}, q_{2}$, then The set of winning coalitions of $H$ will be convex. This grey area is not convex; its convex hull also contains the point corresponding to the only maximal losing coalition $\left\{1,2^{3}\right\}$. This means that the games $H_{1}, \ldots, H_{k}$ cannot be found. What can be done?

Let $P_{1}=\left\{b_{1}, b_{2}\right\}$ and $P_{2}=\left\{c_{1}, c_{2}, c_{3}, c_{4}, c_{5}\right\}$. Consider the two following representations:

First representation. We define two weighted games $G_{1}=\left(P, W_{1}\right)$ and

\footnotetext{
${ }^{2}$ The example we present in this section is the result of an email discussion with Bill Zwicker.
} 
$G_{2}=\left(P, W_{2}\right)$ as follows:

$$
\begin{aligned}
w_{1}\left(b_{1}\right) & =4, w_{1}\left(b_{2}\right)=1.1, \\
\forall_{j \in[5]} & w_{1}\left(c_{j}\right)=1 \text { and quota }=5 .
\end{aligned}
$$

and

$$
\begin{aligned}
w_{1}\left(b_{1}\right) & =1.1, w_{1}\left(b_{2}\right)=4, \\
\forall_{j \in[5]} w_{1}\left(c_{j}\right) & =1 \text { and quota }=5 .
\end{aligned}
$$

Obviously, $H=G_{1} \cap G_{2}$ and, since $H$ is not weighted, we have $\operatorname{dim} H=2$.

Second representation. The above representation failed to represent the 'equivalence' part of $\sim_{H}$ for the players of $P_{1}$, but did succeed with the $P_{2}$ players (in that they did get equal weight for each of the two weightings).

Next, we look at a representation that switches roles: it similarly represents the strict part of $\sim_{H}$ for all players, and it gives the $P_{1}$ players equal weight for each of the weightings (but the five players of $P_{2}$ get different weights). For every subset $X \subset[5]$ such that $|X|=3$ we define a game $G_{X}=\left(P, W_{X}\right)$ by

$$
\begin{aligned}
& w_{X}\left(b_{1}\right)=w_{X}\left(b_{2}\right)=3, \\
& \forall_{i \in X} w_{X}\left(c_{i}\right)=2, \forall_{i \notin X} w_{X}\left(c_{i}\right)=0, \\
& \text { quota }=6
\end{aligned}
$$

There are 10 such sets $X$ of cardinality 3 , and thus 10 weighted games, and it is easy to show that their intersection is the hierarchical game $H$. The nice property of this particular vector of weightings is that it respects the 'strict' part $\sim_{H}$ of the individual desirability order of $H$ in the following sense: $x \prec_{H} y$ iff $w_{X}(x)<w_{X}(y)$ holds for each three-element subset $X$ of [5].

This example explains why the lower bound in the theorem of Freixas and Puente (Theorem 2) had to be reproved. In their proof of the lower bound they allowed only weighted games that assign equal weights to players who are equivalent in the original game. We see that this was not sufficient to claim that the lower bound in their theorem holds.

\section{Boolean dimension of simple games.}

Boolean dimension of a simple game was introduced in Faliszewski et al. (2009). Let $\Phi=\{p, q, \ldots\}$ be a set of propositional variables and let $\mathcal{L}$ denote 
the set of (well-formed) formulas of the first-order propositional logic over $\Phi$ containing only logical connectives $\wedge$ and $\vee^{3}$. For a formula $\phi \in \mathcal{L}$ let $|\phi|$ be the number of variables used to express $\phi$. Suppose also $T$ is a tautology and $\perp$ is a contradiction. Let $G_{i}=\left(P, W_{i}\right), i=1, \ldots, q$, be simple games with the same set of players $P$. We will define the game $G=(P, W)$ by setting for a coalition $C \subseteq P$

$$
C \in W:=\phi\left(C \in W_{1}, \ldots, C \in W_{q}\right)=\top .
$$

We will denote this game $\phi\left(G_{1}, \ldots, G_{q}\right)$. We illustrate this definition with the following simple games which plays an important role in the theory of secret sharing (Beimel et al., 2008). They are called there tripartite.

Example 5. Let $\mathbf{n}=\left(n_{1}, n_{2}, n_{3}\right)$ and $\mathbf{k}=\left(k_{1}, k_{2}, k_{3}\right)$, where $n_{1}, n_{2}, n_{3}$ and $k_{1}, k_{2}, k_{3}$ are positive integers. The game $\Delta_{1}(\mathbf{n}, \mathbf{k})$ is defined on the set $P=P_{1} \cup P_{2} \cup P_{3}$ which is a union of disjoint sets $P_{1}, P_{2}, P_{3}$ of cardinalities $n_{1}, n_{2}, n_{3}$, respectively,. A coalition $C=C_{1} \cup C_{2} \cup C_{3}$, where $C_{i} \subseteq P_{i}$, $i=1,2,3$, is winning iff

$$
\left(\left|C_{1}\right| \geq k_{1}\right) \vee\left[\left(\left|C_{1}\right|+\left|C_{2}\right| \geq k_{2}\right) \wedge\left(\left|C_{1}\right|+\left|C_{2}\right|+\left|C_{3}\right| \geq k_{3}\right)\right]=\top,
$$

where $k_{1}<k_{3}, \quad k_{2}<k_{3}, \quad n_{1} \geq k_{1}, \quad n_{2}>k_{2}-k_{1}$ and $n_{3}>k_{3}-k_{2}$. Obviously, it is organised as $G_{1} \vee\left(G_{2} \wedge G_{3}\right)$, where $G_{1}, G_{2}, G_{3}$ are weighted games.

Definition 7. Let $G$ be a simple game. The smallest positive integer $d$ such that $G$ can be represented as $G=\phi\left(G_{1}, \ldots, G_{n}\right)$, where $G_{1}, \ldots, G_{n}$ are weighted simple games, and $|\phi|=d$ is called the Boolean dimension of $G$.

The Boolean dimension of the game $\Delta_{1}(\mathbf{n}, \mathbf{k})$ from Example 5 is obviously 3 while its classical dimension may (and certainly will) depend on the parameters $\mathbf{n}$ and $\mathbf{k}$. The revised voting rules of the Council of the European Union also has Boolean dimension 3.

Apart from a better ability to reflect the descriptive complexity of games, the Boolean dimension has some nice properties absent in the classical dimension.

\footnotetext{
${ }^{3}$ In paper by Faliszewski et al. (2009) negations were also allowed, however, the authors of that paper considered also non-monotonic simple games which we do not consider.
} 
Proposition 4. The Boolean dimension of a simple game is equal to the Boolean dimension of its dual.

Proof. Let $G_{i}=\left(P, W_{i}\right), i=1,2$, be a simple game and $G_{i}^{*}=\left(P, W_{i}^{*}\right)$, $i=1,2$, be their dual games. Taylor and Zwicker (1999) (see, e.g., Proposition 1.4.3) showed that the following two de Morgan laws are satisfied

$$
\begin{aligned}
& \left(W_{1} \cup W_{2}\right)^{*}=W_{1}^{*} \cap W_{2}^{*}, \\
& \left(W_{1} \cap W_{2}\right)^{*}=W_{1}^{*} \cup W_{2}^{*} .
\end{aligned}
$$

From here it immediately follows that

$$
\begin{aligned}
& \left(G_{1} \vee G_{2}\right)^{*}=G_{1}^{*} \wedge G_{2}^{*}, \\
& \left(G_{1} \wedge G_{2}\right)^{*}=G_{1}^{*} \vee G_{2}^{*},
\end{aligned}
$$

which imply the statement.

However, some features of Boolean dimension are the same as the corresponding features of dimension. In particular, as is the case for dimension, in general the calculation of the exact Boolean dimension of a simple game is NP-hard (Faliszewski et al., 2009).

\section{Conclusion and open questions}

We have answered a question of Freixas and Puente (2008) by showing that the dimension of a complete simple game can grow exponentially in the number of players. The games used to demonstrate this are the disjunctive hierarchical games. We have discussed the pitfalls of the concept of dimension and found some disturbing features of it which led us to reproving and strengthening the theorem of Freixas and Puente about the range in which the dimension of a conjunctive hierarchical game may lie. This research prompts the following questions:

- Can anything be said about the Boolean dimension of a complete game?

- Let $G=(P, W)$ be a complete game with equivalence classes for the desirability relation $P_{1}, \ldots, P_{m}$ so that $P=P_{1} \cup \ldots, \cup P_{m}$. Then for each $i \in[m]$ does there exist a representation of $G$ as an intersection of weighted games such that each game in this representation assigns equal weights to players from $P_{i}$ and respects the strict part of the desirability order $\succeq_{G}$ ? 


\section{Acknowledgements}

Arkadii Slinko was supported by the Royal Society of NZ Marsden Fund UOA-254. He also thanks Bill Zwicker for fruitful discussions of the concept of dimension.

\section{References}

Beimel, A., Tassa, T., Weinreb, E., 2008. Characterizing ideal weighted threshold secret sharing. SIAM J. Discrete Math. 22 (1), 360-397.

Carreras, F., Freixas, J., 1996. Complete simple games. Mathematical Social Sciences 32 (2), 139-155.

Deŭneko, V. G., Woeginger, G. J., 2006. On the dimension of simple monotonic games. European Journal of Operational Research 170 (1), 315-318.

Deng, X., Papadimitriou, C. H., 1994. On the complexity of cooperative solution concepts. Math. Oper. Res. 19 (2), 257-266.

Elgot, C., 1961. Truth functions realizable by single threshold organs. In: Proceedings of the Second Annual Symposium on Switching Circuit Theory and Logical Design. AIEE, pp. 225-245.

Elkind, E., Goldberg, L. A., Goldberg, P. W., Wooldridge, M., 2008. On the dimensionality of voting games. In: Proceedings of the 23rd Conference on Artificial Intelligence. pp. 69-74.

Faliszewski, P., Elkind, E., Wooldridge, M., 2009. Boolean combinations of weighted voting games. In: Proceedings of the 8th International Conference on Autonomous Agents and Multiagent Systems. pp. 185-192.

Freixas, J., 1997. Different ways to represent weighted majority games. Top 5 (2), 201-211.

Freixas, J., 2004. The dimension for the european union council under the nice rules. European Journal of Operational Research 156, 415-419.

Freixas, J., Marciniak, D., 2009. A minimum dimensional class of simple games. Top 17 (2), 407-414. 
Freixas, J., Puente, M. A., 2008. Dimension of complete simple games with minimum. European Journal of Operational Research 188 (2), 555-568.

Gvozdeva, T., Hameed, A., Slinko, A., 2013. Weightedness and structural characterization of hierarchical simple games. Mathematical Social Sciences 65 (3), 181-189.

Hameed, A., Slinko, A., 2015. Roughly weighted hierarchical simple games. International Journal of Game Theory 44 (2), 295-319.

Ieong, S., Shoham, Y., 2005. Marginal contribution nets: A compact representation scheme for coalitional games. In: Proceedings of the 6th ACM Conference on Electronic Commerce. EC '05. ACM, New York, NY, USA, pp. 193-202.

Isbell, J. R., 1958. A class of simple games. Duke Mathematical Journal $25(3), 423-439$.

Kurz, S., Napel, S., 2015. Dimension of the Lisbon voting rules in the EU council: a challenge and new world record. Optimization Letters, 1-12.

Muroga, S., 1971. Threshold Logic and its Applications. Wiley, New York.

Olsen, M., Kurz, S., Molinero, X., 2016. On the Construction of High Dimensional Simple Games. ArXiv:1602.01581.

Peleg, B., 2002. Game-theoretic analysis of voting in committees. Handbook of social choice and welfare 1, 395-423.

Ramamurthy, K., 2012. Coherent structures and simple games. Vol. 6. Springer Science \& Business Media.

Simmons, G. J., 1990. How to (really) share a secret. In: Proceedings of the 8th Annual International Cryptology Conference on Advances in Cryptology. Springer-Verlag, pp. 390-448.

Tassa, T., 2007. Hierarchical threshold secret sharing. Journal of Cryptology 20 (2), 237-264.

Taylor, A., Zwicker, W., 1993. Weighted voting, multicameral representation, and power. Games and Economic Behavior 5 (1), 170-181. 
Taylor, A. D., Zwicker, W. S., 1999. Simple Games: Desirability Relations, Trading, Pseudoweightings. Princeton University Press.

von Neumann, J., Morgenstern, O., 1944. Theory of Games and Economic Behavior. Princeton University Press. 